\title{
Análise da inserção das tecnologias de prototipagem e fabricação digital nos cursos de Design de duas IES do estado de São Paulo
}

Analysis of the insertion of the technologies of prototyping and digital fabrication in the courses of Design of two IES of the state of São Paulo.

SILVA, Conrado Renan da; Mestrando em Design; FAAC - Universidade Estadual Paulista

conrado@faac.unesp.br

BARATA, Tomas Queiroz Ferreira; Doutor; FAAC - Universidade Estadual Paulista

barata@faac.unesp.br

\section{Resumo}

As tecnologias de prototipagem rápida têm contribuído para o desenvolvimento de produtos, devido às possibilidades de produzir modelos e protótipos num ciclo de tempo reduzido. Quando inseridas no ambiente universitário, podem transformar os métodos de ensino e aprendizagem e aproximar os alunos às necessidades do mercado. Este estudo apresenta uma análise da inserção das tecnologias de prototipagem rápida em duas instituições de ensino superior do estado de São Paulo com o intuito de comparar as atividades projetivas nos cursos de graduação de design. A metodologia adotada no trabalho se organiza em duas etapas, o levantamento teórico sobre prototipagem rápida e fabricação digital e análise de projetos políticos pedagógicos sobre a inserção das tecnologias de prototipagem rápida nos cursos de graduação. Como resultado, são apresentados dados comparativos entre os cursos de Design das instituições visando compreender como cada curso trata a relação da inserção das tecnologias de prototipagem nas atividades de ensino.

Palavras Chave: Prototipagem Rápida; Fabricação Digital; Ensino do Design e IES.

\begin{abstract}
Rapid prototyping technologies have contributed to product development due to the possibilities of producing models and prototypes in a reduced time cycle. When inserted in the university environment, they can transform teaching and learning methods and bring students closer to market needs. This study presents an analysis of the insertion of rapid prototyping technologies in two higher education institutions of the state of São Paulo in order to compare the projective activities in the graduation courses of design. The methodology adopted in the work is organized in two stages, the theoretical survey on rapid prototyping and digital fabrication and analysis of pedagogical political projects on the insertion of rapid prototyping technologies in undergraduate courses. As a result, comparative data are presented among the Design courses of the institutions aiming to understand how each course deals with the relation of the insertion of prototyping technologies in teaching activities.
\end{abstract}

Keywords: Rapid Prototyping; Digital Manufacturing; Education of Design and IES. 


\section{Introdução}

As tecnologias de Prototipagem Rápida (PR) têm estado cada vez mais presentes nas Instituições de Ensino Superior (IES), ampliando as possibilidades de ensino e pesquisa para os alunos de Design e áreas correlatas como Arquitetura e Engenharias, principalmente nas atividades didático-pedagógicas relacionadas com o desenvolvimento de projetos e produtos. Essas tecnologias têm contribuído de maneira significativa nos Processos de Desenvolvimento de Produtos (PDP), devido às possibilidades de produzir modelos e protótipos de maneira rápida, eficaz e precisa, permitindo que o profissional execute testes, faça ajustes e elabore protótipos de um produto num ciclo de tempo reduzido.

A inserção das tecnologias PR nos ambientes universitários se dá, devido à facilidade em produzir modelos e protótipos a partir de desenhos assistidos por computador CAD (Computer Aided Design) e convertendo as informações para o ambiente CAM (Computer Aided Manufacturing). É útil desde a concepção do protótipo digital, até a elaboração do produto físico, tornando as ideias do designer palpáveis, sendo um recurso para materializá-las, fato que facilita a visualização e compreensão do produto, permite a conferência de encaixes e componentes, auxilia na verificação de dimensões e contribui para validar aspectos relacionados a ergonomia do produto (ALENCAR; BÁRTOLO, 2012).

Além disso, nos cursos superiores de design, essa relação com a tecnologia potencializa o PDP e o entendimento dos alunos, pois de acordo com Volpato et al (2007), a maioria dos profissionais, independente de gênero ou classe social, tende a assimilar a informação projetiva de forma mais rápida, quando é transmitida por um modelo físico e não apenas por desenhos ou ilustrações bidimensionais, isso ocorre porque o modelo físico permite interações.

Bürdek (1994) afirma que, outro fato significativo é o crescente número de indústrias que têm adquirido maquinários de produção controlados por computador (CNC), o que leva também a uma transformação nos modos de produção, construção e design. Os sistemas CAD transformaram a atividade projetiva, os dados geométricos gerados uma vez, permitem rapidamente modificações de detalhes, encaixes, variantes de cores, alinhamentos entre outros aspectos, tornando o PDP bem mais ágil e preciso.

Diante desse contexto, conclui-se que as tecnologias de prototipagem digital trazem diversos benefícios no processo de desenvolvimento de produtos e quando inseridas no ambiente universitário, podem transformar os métodos de ensino e aprendizagem de projetos, potencializando os processos criativos e, da mesma forma, aproximam os alunos às necessidades do mercado. Para tanto, a abordagem deste trabalho visa a analisar a inserção das tecnologias de PR disponíveis em duas instituições de ensino superior (IES) do estado de São Paulo com o intuito de comparar as atividades do ensino de cursos de graduação de Design e compreender como tem sido as práticas pedagógicas em disciplinas projetivas. Para isso, a metodologia aplicada na pesquisa se pautou em dois aspectos: a) realização de levantamento teórico sobre prototipagem rápida e fabricação digital e suas características e; b) análise dos Projetos Políticos Pedagógicos (PPP) sobre a inserção das novas tecnologias de prototipagem rápida nos cursos de graduação em Design. 


\section{Revisão bibliográfica}

A revisão bibliográfica para o desenvolvimento deste estudo abordou os conceitos relacionados às tecnologias de prototipagem rápida e a inserção no ambiente universitário, visando compreender a importância de tais tecnologias nas atividades de ensino do design, através dos seguintes tópicos: a) Novas tecnologias e o ensino do design; b) Prototipagem rápida e fabricação digital e; c) Tecnologias disponíveis para o desenvolvimento de produto.

\subsection{Novas tecnologias e o ensino do Design}

As modificações do ensino na contemporaneidade estão diretamente relacionadas ao uso de novas tecnologias. Ao refletir sobre design e tecnologia, Moura (2005) apresenta as relações existentes, afirmando que design é tecnologia e que a tecnologia somente não traduz plenamente a definição de design, mas que a utiliza para se obter novos resultados de projetos, sendo assim, design é o campo, a área por excelência que surge a partir da relação com a tecnologia.

O uso das tecnologias de prototipagem no ambiente universitário aproxima o aluno à realidade do mercado de trabalho e permitem uma maior flexibilidade no desenvolvimento de projetos, ou seja, acredita-se que as atividades de ensino e aprendizagem são potencializadas com emprego das novas tecnologias de fabricação rápida (PORTUGAL, 2016). Segundo Lesko (2012), entre os benefícios que as PR's apresentam, a redução nos tempos de desenvolvimento do produto é a que mais se destaca, ainda mais nas condições atuais de competitividade do mercado, ou seja, fazer com que um produto seja lançado mais depressa é uma grande vantagem.

Ashby e Johnson (2011) complementam que, novos processos produtivos e materiais podem ser inspiradores para os designers e que a linguagem das novas tecnologias parece despertar uma percepção do novo e de algo melhor. A utilização dessas tecnologias de prototipagem rápida e fabricação digital pelas IES tem um papel decisivo e abre um campo enorme de possibilidades onde o estudante de design poderá atuar. Segundo Bruscato et al. (2014), os protótipos podem ser utilizados para estudos, análises, avaliações de alternativas e até mesmo para apresentações finais de projeto.

Lesko (2012) fala acerca de outros benefícios que as PR's podem proporcionar, dentre eles é a disponibilidade de máquinas compactas e de baixo custo que permitem que os designers e profissionais da área tenham acesso a esse tipo de tecnologia de modo fácil, permitindo inclusive que possuam em casa ou escritório. As possibilidades de atuação de um designer são diversas e com o advento das novas tecnologias a partir dos anos noventa, elas se tornaram ainda maiores. Segundo Portugal (2013), "o fortalecimento do design brasileiro vem trilhando esse caminho com ações inter-relacionadas referentes ao campo profissional e ao mercado de trabalho e, especialmente, as questões do ensino e da pesquisa em design".

\subsection{Prototipagem Rápida e Fabricação Digital}

A prototipagem rápida tem como fundamento a construção de um modelo físico a partir da concepção de um modelo produzido virtualmente (PV-protótipo virtual) auxiliado por computador 
(CAD). Segundo Groover (2014), o desenvolvimento de uma variedade de tecnologias de PR, foi devido à necessidade dos projetistas terem em mãos o modelo físico e não apenas um modelo virtual, pois desta forma poderiam examinar visual e fisicamente a peça e realizar testes.

A Fabricação Digital (FD) pode ser compreendida como uma alternativa para a produção em série, através da manufatura local e customizada, constitui-se como a fase final da etapa de criação. O protótipo virtual é produzido por equipamentos controlados por computador, podendo ser executados por mecanismos baseados em tecnologia de adição ou de subtração de material (SALVAN PAGNAN; MOTTIN, 2018).

Bruscato et al.,(2014), complementa ao afirmar que tanto no processo aditivo quanto no subtrativo, a primeira etapa é a criação de um modelo virtual a partir de um software CAD. De acordo com Salvan Pagnan e Mottin (2018), a tecnologia CAD possibilita projetar bi e tridimensionalmente através de softwares específicos e a tecnologia CAM interpreta as informações que são processadas por máquinas de CNC que são capazes de materializar os projetos.

Desta maneira, as tecnologias de prototipagem e fabricação digital, tornam-se uma excelente ferramenta para a compreensão do processo de desenvolvimento de produtos que de acordo com Löbach (2001) vai desde a definição do problema, levantamento de informações sobre o problema, a criação de alternativas e o desenvolvimento da melhor alternativa que pode ser um produto. Essas tecnologias de PR e FD tornam-se muito importantes na compreensão dos alunos ao desenvolver projetos.

\subsection{Tecnologias disponíveis para o desenvolvimento de produto}

A utilização de tecnologias contemporâneas de fabricação para o desenvolvimento de protótipos, como as fresadoras CNC, o corte a laser e a Prototipagem Rápida que inclui as impressoras $3 \mathrm{~d}$ nos seus mais variados formatos e as linguagens instrumentais tecnológicas projetivas envolvidas, aponta uma aproximação de estudantes e profissionais de Design com as necessidades requeridas pelas indústrias (BARBOSA, 2009). O uso das dessas tecnologias no ambiente universitário pode transformar os métodos de ensino e aprendizagem e facilitar o entendimento do aluno no PDP.

A Prototipagem por Comando Numérico por Computador (CNC) é uma tecnologia de fabricação automatizada, que possui precisão através da remoção de materiais até a obtenção da peça desejada. Segundo Groover (2014), "é a forma de automação programável em que as atuações mecânicas de um equipamento são controladas por um programa contendo códigos alfanuméricos." A execução é feita for equipamentos de três eixos automatizados (fresadora ou tornos) ou por equipamentos de oito eixos (usinagem) que esculpem a forma desejada em diversos tipos de materiais (BARBOSA, 2009).

O Corte a Laser é obtido através de máquinas com três eixos ( $x, y$ e eixo $Z$ que possui regulagem), as máquinas de corte a laser possuem um tubo de Dióxido de Carbono $\left(\mathrm{CO}^{2}\right)$ que emite o raio laser podendo variar de 60 à 150 Watts, que é determinado pelas dimensões da máquina e a finalidade do trabalho a ser cortado, bem como materiais, espessuras, dentre outros. 
A tecnologia do corte a laser permite a gravação e recorte de desenhos através da queima do material pelo raio laser, a obtenção pode ser feita através de vetores obtidos por meios do sistema CAD (BARBOSA, 2009).

As tecnologias de Prototipagem Rápida (PR) baseiam-se na construção de modelos e protótipos por adição ou subtração de material. Nesta tecnologia, o projeto é modelado em sistemas CAD por softwares 3D e, quando levada ao software específico da máquina, esse arquivo é fatiado em camadas e posteriormente é feita a construção do protótipo empilhando-se uma camada sobre a outra, esse processo é conhecido por deposição de camadas.

\section{Materiais e Métodos}

O método de pesquisa utilizado neste trabalho é de caráter teórico e comparativo, este é indicado especialmente quando se deseja verificar similaridades e divergências em um estudo descritivo (LAKATOS; MARCONI,2001). O método adotado possui as seguintes etapas:

a) Realização de levantamento teórico - Pesquisa de fundamentação teórica conceitual com o objetivo de caracterizar os conceitos de Prototipagem e Fabricação Digital, através da consulta de livros, teses, artigos e sites;

b) Investigação dos Projetos Políticos Pedagógicos (PPP) - Levantamento de dados sobre o perfil dos cursos de graduação em Design de duas Universidades Públicas do Estado de São Paulo, aferindo grade curricular e ementas das disciplinas, visando a relação delas com as tecnologias de PR e FD;

c) Definição de palavras-chaves para direcionar a pesquisa nos PPP's das duas IES;

d) Análise dos dados, considerando: a) identificação das disciplinas que tenham relação direta com PR e FD através dos descritivos dos planos de ensino e ementas de disciplinas, relacionadas com palavras-chaves pré-determinadas e; b) Identificação das disciplinas que possuam relação indireta com PR e FD na busca de palavras-chaves nos descritivos dos planos de ensino e ementas, considerando aquelas que se relacionem devido a interdisciplinaridade e integração das disciplinas projetivas;

e) Elaboração de um gráfico comparativo das disciplinas e carga horária das duas IES;

\section{Resultados e discussões}

Os perfis dos cursos foram levantados, a partir do acesso aos Projetos Políticos Pedagógicos disponível no site das instituições, para essa pesquisa foram considerados, os cursos de Design de duas IES públicas do estado de São Paulo. A consulta a esses PPP's resultou num levantamento de dados específicos de cada curso, das grades curriculares, planos de ensino, carga horária, ementas das disciplinas. As informações referentes às disciplinas são apresentadas a partir de uma seleção daquelas que possuem relação direta ou indireta com as tecnologias de prototipagem e fabricação digital. 


\subsection{Análise do curso da IES 1}

O curso da IES 1, é composto por disciplinas base e disciplinas de ênfases estruturadas (quadro 1) que possibilitam ao aluno a escolha de uma especialidade, podendo ser: Design Digital, Design Gráfico, Design de Moda e Design de Produto.

Quadro 1: Grade Curricular do curso de design da IES 1

\begin{tabular}{|c|c|c|c|}
\hline 10 Semestre & 20 Semestre & 3 o Semestre & 4은 Semestre \\
\hline Projeto $1-60 \mathrm{~h}$ & Projeto 2 - 60h & Projeto $3-60 \mathrm{~h}$ & Projeto $4-60 \mathrm{~h}$ \\
\hline Plástica 1 - 60h & Plástica 2 - 30h & Ilustração - 120h & Desenho Técnico 1 - 30h \\
\hline $\begin{array}{c}\text { Desenho de Observação } 1 \text { - } \\
60 \mathrm{~h} \\
\end{array}$ & $\begin{array}{c}\text { Desenho de Observação } \\
2-30 \mathrm{~h} \\
\end{array}$ & $\begin{array}{c}\text { Design e Cultura na } \\
\text { história Gráfica - 30h }\end{array}$ & Sociologia - 30h \\
\hline $\begin{array}{l}\text { Fotografia e Discurso } \\
\text { Multimodal - } 60 \mathrm{~h} \\
\end{array}$ & $\begin{array}{l}\text { Design e Cultura no } \\
\text { Modernismo - } 60 \mathrm{~h}\end{array}$ & $\begin{array}{c}\text { Psicologia: Cognição e } \\
\text { Percepção no Design 30h }\end{array}$ & Filosofia - 30h \\
\hline $\begin{array}{c}\text { Design e Cultura na } \\
\text { Contemporaneidade }-60 \mathrm{~h}\end{array}$ & $\begin{array}{c}\text { Semiótica no Design } \\
-60 \mathrm{~h} \\
\end{array}$ & $\begin{array}{c}\text { Design e Complexidade } \\
60 \mathrm{~h} \\
\end{array}$ & $\begin{array}{l}\text { Ateliê de Madeira } \\
60 \mathrm{~h} \\
\end{array}$ \\
\hline $\begin{array}{l}\text { Design: Materiais e } \\
\text { Processos } 1-60 \mathrm{~h}\end{array}$ & $\begin{array}{c}\text { Design: Materiais e } \\
\text { Processos } 2 \text { - 30h } \\
\text { Processos de Fabricação } \\
30 \mathrm{~h}\end{array}$ & $\begin{array}{l}\text { Planejamento Gráfico e } \\
\text { Tipográfico - 60h }\end{array}$ & $\begin{array}{c}\text { Modelagem e } \\
\text { Prototipagem } \\
60 \mathrm{~h}\end{array}$ \\
\hline
\end{tabular}

\begin{tabular}{|c|c|c|c|}
\hline 50 Semestre & 60 Semestre & 7옹 Semestre & $8^{\circ}$ Semestre \\
\hline Projeto 5 - 90h & Projeto 6 - 90h & Projeto 7 - 120h & Projeto 8 - $150 \mathrm{~h}$ \\
\hline Desenho Técnico 2 - 90h & $\begin{array}{c}\text { Processos Criativos e } \\
\text { Expressivos- } 60 \mathrm{~h} \\
\end{array}$ & $\begin{array}{l}\text { Direitos Humanos, Ética e } \\
\text { Acessibilidade- } 60 \mathrm{~h} \\
\end{array}$ & $\begin{array}{c}\text { Empreendedorismo e } \\
\text { Gestão em Design - 60h }\end{array}$ \\
\hline $\begin{array}{c}\text { Antropologia Cultural e } \\
\text { Design }-60 \mathrm{~h}\end{array}$ & $\begin{array}{c}\text { Conhecimento Científico } \\
30 \mathrm{~h}\end{array}$ & $\begin{array}{c}\begin{array}{c}\text { Comunicação em } \\
\text { Marketing - 30h }\end{array} \\
\text { Design de Serviços 30h }\end{array}$ & \\
\hline $\begin{array}{l}\text { Ergonomia aplicada ao } \\
\text { Design- } 60 \mathrm{~h}\end{array}$ & $\begin{array}{c}\text { Design e Experiência do } \\
\text { Usuário - 60h }\end{array}$ & $\begin{array}{c}\text { Design Inclusivo - 30h } \\
\text { Tecnologias Assistivas } \\
30 \mathrm{~h}\end{array}$ & \\
\hline & $\begin{array}{c}\text { Design para a } \\
\text { Sustentabilidade }-30 \mathrm{~h}\end{array}$ & $\begin{array}{c}\text { Design da Informação } \\
30 \mathrm{~h} \\
\end{array}$ & \\
\hline
\end{tabular}

Fonte: Elaborado pelos autores

A grade curricular da IES 1 é composta por disciplinas gerais e disciplinas optativas, que compõe uma carga horária total de 3420 horas-aula ao longo de 8 semestres, sendo subdivididas nos seguintes eixos: Projetual, Expressivo, Cultural, Tecnológico e Mercado. Esses eixos estruturantes de formação estruturam e conferem uma lógica ao conjunto de disciplinas obrigatórias e optativas, às atividades complementares, à avaliação e a promoção da interdisciplinaridade.

As disciplinas de ênfase são estruturadas por meio de disciplinas que possibilitam ao aluno 
a aquisição de uma das especialidades oferecidas ou de realizar uma ou mais disciplinas de ênfase visando ampliar seu repertório de conhecimentos para a prática profissional.

Quadro 2: Disciplinas de ênfases do curso de design da IES 1

\begin{tabular}{|c|c|c|c|}
\hline DIGITAL & GRÁFICO & MODA & PRODUTO \\
\hline $\begin{array}{c}\text { Introdução à lógica de } \\
\text { programação e } \\
\text { cultura hacker - 60h }\end{array}$ & Tipografia 1 60h & Superfícies Têxteis - 60h & $\begin{array}{c}\text { Prototipagem e Processos de } \\
\text { Fabricação Digital 3D - 60h }\end{array}$ \\
\hline $\begin{array}{c}\text { Design Paramétrico e } \\
\text { Linguagens Emergentes } \\
-60 \mathrm{~h}\end{array}$ & $\begin{array}{c}\text { Design } \\
\text { Corporativo - 60h }\end{array}$ & Modelagem - 60h & $\begin{array}{c}\text { Modelagem Avançada em 3D } \\
-60 \mathrm{~h}\end{array}$ \\
\hline $\begin{array}{c}\text { Laboratório de Mídias } \\
\text { Interativas - 60h }\end{array}$ & Tipografia 2 - 60h & $\begin{array}{c}\text { Processos Produtivos em } \\
\text { Moda - 60h }\end{array}$ & Design de Objetos - 60h \\
\hline $\begin{array}{c}\text { Ludicidade e Design } \\
\text { de Jogos - 60h }\end{array}$ & $\begin{array}{c}\text { Produção Gráfica } \\
-60 \mathrm{~h}\end{array}$ & $\begin{array}{c}\text { Design de Acessórios - } \\
60 \mathrm{~h}\end{array}$ & Design de Mobiliário - 60h \\
\hline Mídia, Corpo e \\
Tecnologia -60h & $\begin{array}{c}\text { Design Editorial - } \\
60 \mathrm{~h}\end{array}$ & $\begin{array}{c}\text { Joalheria Contemporânea } \\
-60 \mathrm{~h}\end{array}$ & Design de Ambientes - 60h \\
\hline $\begin{array}{c}\text { Open design e } \\
\text { cultura maker - 60h }\end{array}$ & $\begin{array}{c}\text { Fotografia } \\
-60 \mathrm{~h}\end{array}$ & $\begin{array}{c}\text { Desenvolvimento de } \\
\text { Coleções - 60h }\end{array}$ & Laboratório de Metais - 60h \\
\hline
\end{tabular}

Fonte: Elaborado pelos autores

\subsection{Análise do curso da IES 2}

A quadro 3 apresenta a grade curricular do curso de design da IES 2 com os nomes das disciplinas e suas respectivas cargas horárias, o curso é composto por disciplinas base e disciplinas eletivas optativas.

Quadro 3: Grade Curricular do curso de design da IES 2

\begin{tabular}{|c|c|c|c|}
\hline 10 Semestre & 20 Semestre & 30 Semestre & 4ㅇ Semestre \\
\hline $\begin{array}{c}\text { Linguagens Visuais - } \\
90 \mathrm{~h} \\
\end{array}$ & $\begin{array}{l}\text { Modelos e protótipos } \\
\text { em Design - 90h }\end{array}$ & $\begin{array}{l}\text { Introdução ao projeto } \\
\text { visual }-90 \mathrm{~h} \\
\end{array}$ & $\begin{array}{c}\text { Produção Gráfica - } \\
90 \mathrm{~h}\end{array}$ \\
\hline $\begin{array}{l}\text { História da arte, da } \\
\text { arquitetura, e suas } \\
\text { técnicas }-60 \mathrm{~h}\end{array}$ & $\begin{array}{l}\text { Fundamentos Sociais } \\
\text { do Design }-30 \mathrm{~h}\end{array}$ & $\begin{array}{c}\text { Consumo na } \\
\text { contemporaneidade - } \\
30 \mathrm{~h}\end{array}$ & $\begin{array}{c}\text { Projeto Integrado - } \\
180 \mathrm{~h}\end{array}$ \\
\hline $\begin{array}{l}\text { Fundamentos do } \\
\text { Projeto: }\end{array}$ & $\begin{array}{c}\text { História do Design I- } \\
30 \mathrm{~h}\end{array}$ & \multirow{2}{*}{$\begin{array}{l}\text { História do Design II } \\
\text { 30h }\end{array}$} & \multirow[b]{2}{*}{ Ergonomia I - 30h } \\
\hline $\begin{array}{l}\text { Experimentação e } \\
\text { Materialidade - 90h }\end{array}$ & $\begin{array}{c}\text { Estratégias } \\
\text { Analógicas de Projeto } \\
-90 \mathrm{~h}\end{array}$ & & \\
\hline Tipografia $-90 \mathrm{~h}$ & $\begin{array}{l}\text { Laboratório da } \\
\text { imagem - 90h }\end{array}$ & $\begin{array}{c}\text { Introdução design de } \\
\text { serviços }-90 \mathrm{~h} \\
\end{array}$ & $\begin{array}{l}\text { História do Design III } \\
-30 \mathrm{~h} \\
\end{array}$ \\
\hline $\begin{array}{l}\text { Materiais e processos } \\
\text { de produção I - } 60 \mathrm{~h}\end{array}$ & $\begin{array}{l}\text { Materiais e processos } \\
\text { de produção II - } 60 \mathrm{~h}\end{array}$ & $\begin{array}{l}\text { Introdução ao projeto } \\
\text { de produto }-90 \mathrm{~h}\end{array}$ & $\begin{array}{c}\text { Linguagem } \\
\text { computacional }-60 \mathrm{~h}\end{array}$ \\
\hline $\begin{array}{c}\text { Técnicas de } \\
\text { visualização e } \\
\text { representação I - 60h }\end{array}$ & $\begin{array}{c}\text { Técnicas de } \\
\text { visualização e } \\
\text { representação II -60h }\end{array}$ & $\begin{array}{l}\text { Metodologia em projeto } \\
\text { em Design }-60 \mathrm{~h}\end{array}$ & \\
\hline
\end{tabular}




\begin{tabular}{|c|c|c|c|}
\hline 50 Semestre & 60 Semestre & 70 Semestre & 80 Semestre \\
\hline $\begin{array}{c}\text { 2x Optativas eletivas } \\
\text { de projeto MIP/MOP } \\
(1 \text { por bimestre })- \\
90 \mathrm{~h} \\
\end{array}$ & $\begin{array}{l}\text { 2x Optativas eletivas de } \\
\text { projeto MIP/MOP ( } 1 \\
\text { por bimestre })-90 \mathrm{~h}\end{array}$ & $\begin{array}{c}\text { 2x Optativas eletivas } \\
\text { de projeto MIP/MOP ( } 1 \\
\text { por bimestre) - } 90 \mathrm{~h}\end{array}$ & $\begin{array}{c}\text { 2x Optativas eletivas } \\
\text { de projeto MIP/MOP } \\
\text { (1 por bimestre) - } \\
90 \mathrm{~h}\end{array}$ \\
\hline $\begin{array}{l}\text { História do Design IV } \\
30 \mathrm{~h}\end{array}$ & $\begin{array}{c}\text { Cultura Urbana na } \\
\text { contemporaneidade - } \\
60 \mathrm{~h}\end{array}$ & $\begin{array}{c}\text { Optativa eletiva } 4 \\
\text { créditos - 60h ou } 2 \mathrm{x} \\
\text { Optativa eletivas } 2 \\
\text { créditos }-30 \mathrm{~h} \\
\end{array}$ & $\begin{array}{c}\text { Legislação, normas e } \\
\text { ética profissional - } \\
\text { 30h }\end{array}$ \\
\hline Ergonomia II - 30h & $\begin{array}{c}\text { 2x Optativas eletivas de } \\
\text { projeto MIP/MOP (1 } \\
\text { por bimestre) }-90 \mathrm{~h}\end{array}$ & $\begin{array}{l}\text { 2x Optativas eletivas } \\
\text { de projeto MIP/MOP ( } 1 \\
\text { por bimestre) }-90 \mathrm{~h}\end{array}$ & $\begin{array}{c}\text { Textos } \\
\text { comunicacionais } \\
30 \mathrm{~h} \\
\end{array}$ \\
\hline $\begin{array}{l}\text { 2x Optativas eletivas } \\
\text { de projeto MIP/MOP } \\
\text { (1 por bimestre) }-90 \mathrm{~h}\end{array}$ & $\begin{array}{l}\text { Meios e métodos de } \\
\text { produção - } 60 \mathrm{~h}\end{array}$ & $\begin{array}{c}\text { Design para a } \\
\text { comunicação } \\
60 \mathrm{~h}\end{array}$ & $\begin{array}{c}\text { 2x Optativas eletivas } \\
\text { de projeto MIP/MOP } \\
\text { (1 por bimestre) - } \\
90 \mathrm{~h}\end{array}$ \\
\hline $\begin{array}{c}\text { Gestão de Marcas - } \\
30 \mathrm{~h}\end{array}$ & $\begin{array}{l}\text { Optativa eletiva } 4 \\
\text { créditos - 60h ou } 2 \mathrm{x} \\
\text { Optativa eletivas } 2 \\
\text { créditos }-30 \mathrm{~h}\end{array}$ & $\begin{array}{c}\text { Viabilidade e gestão de } \\
\text { projetos em Design - } \\
30 \mathrm{~h}\end{array}$ & $\begin{array}{l}\text { Práticas audiovisuais } \\
-90 \mathrm{~h}\end{array}$ \\
\hline $\begin{array}{c}\text { Computação } \\
\text { Interativa - 30h }\end{array}$ & & $\begin{array}{c}\text { Inovação e } \\
\text { empreendedorismo - } \\
30 \mathrm{~h}\end{array}$ & $\begin{array}{l}\text { Optativa eletiva/ } \\
\text { LIVRE de } 4 \text { créditos } \\
\text { 60h ou 2x Optativas } \\
\text { eletivas/ LIVRE } 2 \\
\text { créditos - } 30 \mathrm{~h} \\
\end{array}$ \\
\hline $\begin{array}{c}\text { Design, ambiente e } \\
\text { sustentabilidade - } \\
30 \mathrm{~h}\end{array}$ & & & $\begin{array}{l}\text { Optativa eletiva/ } \\
\text { LIVRE de } 4 \text { créditos } \\
\text { 60h ou 2x Optativas } \\
\text { eletivas / LIVRE } 2 \\
\text { créditos - } 30 \mathrm{~h}\end{array}$ \\
\hline \multicolumn{4}{|l|}{$\begin{array}{c}\text { Teorias do Signo - } \\
30 \mathrm{~h} \\
\end{array}$} \\
\hline 9o Semestre & TCC II - 270h & $\begin{array}{c}\text { Optativa eletiva } 4 \\
\text { créditos - 60h ou } 2 \mathrm{x} \\
\text { Optativas eletivas } 2 \\
\text { créditos }-30 \mathrm{~h}\end{array}$ & $\begin{array}{l}\text { Optativa eletiva/ } \\
\text { LIVRE de } 4 \text { créditos - } \\
\text { 60h ou } 2 \text { x optativas } \\
\text { eletivas/LIVRE } 2 \\
\text { créditos - } 30 \mathrm{~h}\end{array}$ \\
\hline $10^{\circ}$ Semestre & TCC III - 300h & $\begin{array}{c}\text { Atividades } \\
\text { complementares em } \\
\text { design } \\
\end{array}$ & \\
\hline
\end{tabular}

Fonte: Elaborado pelos autores

A grade curricular é generalista e possui disciplinas optativas eletivas, optativas modulares de projeto (MOP) e Módulos Interdepartamentais de Projeto (MIP), compondo uma carga horária total de 3840 horas ao longo de 10 semestres, o curso não possui definição de habilitações específicas. As disciplinas optativas abordam diversas temáticas que permitem ao aluno um enfoque numa determinada área que mais se agrade. 
Quadro 4: Disciplinas Optativas do curso de design da IES 2

\begin{tabular}{|c|c|c|c|c|c|}
\hline Disciplinas & $\begin{array}{c}\text { Optativas } \\
\text { Eletivas do } \\
\text { Curso }\end{array}$ & Disciplinas & $\begin{array}{c}\text { MOP - } \\
\text { Módulo } \\
\text { Optativo de } \\
\text { Projeto } \\
\end{array}$ & Disciplinas & $\begin{array}{c}\text { MIP - Módulo } \\
\text { Interdepartamental } \\
\text { de Projeto }\end{array}$ \\
\hline $\begin{array}{c}\text { História da } \\
\text { tecnologia } \\
\text { séculos XVII ao } \\
\text { XXI }\end{array}$ & $30 h$ & $\begin{array}{l}\text { MOP: Design } \\
\text { para } \\
\text { mobilidade e } \\
\text { transporte }\end{array}$ & $90 \mathrm{~h}$ & $\begin{array}{c}\text { MIP: } \\
\text { Design, } \\
\text { materiais e } \\
\text { produção }\end{array}$ & $90 h$ \\
\hline $\begin{array}{c}\text { Estudos } \\
\text { dirigidos em } \\
\text { história, crítica } \\
\text { e projeto de } \\
\text { Design }\end{array}$ & $60 \mathrm{~h}$ & $\begin{array}{l}\text { MOP: Humor } \\
\text { no design }\end{array}$ & $90 \mathrm{~h}$ & $\begin{array}{l}\text { MIP: } \\
\text { Design, } \\
\text { história e } \\
\text { memória }\end{array}$ & $90 h$ \\
\hline $\begin{array}{l}\text { Processos de } \\
\text { criação e Design } \\
\text { visual }\end{array}$ & $60 \mathrm{~h}$ & $\begin{array}{l}\text { MOP: Design } \\
\text { para usuários } \\
\text { específicos }\end{array}$ & $90 \mathrm{~h}$ & \begin{tabular}{|c|} 
MIP: \\
Animação e \\
artes do \\
vídeo \\
\end{tabular} & $90 h$ \\
\hline $\begin{array}{l}\text { Teoria do } \\
\text { Design }\end{array}$ & $60 \mathrm{~h}$ & $\begin{array}{l}\text { MOP: } \\
\text { Laboratório do } \\
\text { futuro }\end{array}$ & $90 \mathrm{~h}$ & \begin{tabular}{|c|} 
MIP: Design \\
para \\
ambientes \\
digitais \\
\end{tabular} & $90 h$ \\
\hline $\begin{array}{l}\text { Crítica do } \\
\text { Design }\end{array}$ & $60 \mathrm{~h}$ & $\begin{array}{l}\text { MOP: Design e } \\
\text { biomimética }\end{array}$ & $90 \mathrm{~h}$ & & \\
\hline Design de Tipos & $60 \mathrm{~h}$ & $\begin{array}{l}\text { MOP: Design } \\
\text { para o Lazer }\end{array}$ & $90 \mathrm{~h}$ & & \\
\hline $\begin{array}{l}\text { Usabilidade e } \\
\text { desempenho }\end{array}$ & $30 \mathrm{~h}$ & $\begin{array}{l}\text { MOP: Design, } \\
\text { espaço e } \\
\text { ambientação }\end{array}$ & $90 \mathrm{~h}$ & & \\
\hline $\begin{array}{l}\text { Design, cultura } \\
\text { e materialidade }\end{array}$ & $60 \mathrm{~h}$ & $\begin{array}{l}\text { MOP: Design } \\
\text { de unidades, } \\
\text { famílias e } \\
\text { sistemas } \\
\end{array}$ & $90 \mathrm{~h}$ & & \\
\hline $\begin{array}{c}\text { Desenho } \\
\text { paramétrico e } \\
\text { fabricação } \\
\text { digital }\end{array}$ & $60 \mathrm{~h}$ & $\begin{array}{l}\text { MOP: Design } \\
\text { para saúde e } \\
\text { bem-estar }\end{array}$ & $90 \mathrm{~h}$ & & \\
\hline $\begin{array}{l}\text { Tecnologia da } \\
\text { computação } \\
\text { gráfica } \\
\end{array}$ & $60 \mathrm{~h}$ & $\begin{array}{l}\text { MOP: Design } \\
\text { experimental }\end{array}$ & $90 \mathrm{~h}$ & & \\
\hline $\begin{array}{c}\text { Design e } \\
\text { programação } \\
\text { de games } \\
\end{array}$ & $60 \mathrm{~h}$ & $\begin{array}{c}\text { MOP: Design } \\
\text { da informação }\end{array}$ & $90 \mathrm{~h}$ & & \\
\hline \multirow[t]{2}{*}{$\begin{array}{c}\text { Teoria e análise } \\
\text { das mídias } \\
\text { audiovisuais } \\
\end{array}$} & $30 \mathrm{~h}$ & $\begin{array}{l}\text { MOP: Design } \\
\text { editorial }\end{array}$ & $90 \mathrm{~h}$ & & \\
\hline & & $\begin{array}{l}\text { MOP: Livro e } \\
\text { narrativas } \\
\text { visuais }\end{array}$ & $90 \mathrm{~h}$ & & \\
\hline
\end{tabular}

Fonte: Elaborado pelos autores. 


\subsection{Palavras-Chaves para a pesquisa}

Para fazer a seleção das disciplinas que possuem relação direta e indireta com as tecnologias de prototipagem e fabricação digital, foram eleitas algumas palavras-chaves para nortear a pesquisa nos PPP's das duas IES conforme a tabela abaixo.

Tabela 1: Palavras-chaves

\begin{tabular}{|c|l|}
\hline & Fabricação; Tecnologias; Processos de fabricação; Impressão 3d; produção e transformação no \\
PALAVRAS- & PDP; 3D; Printing; Protótipos/Modelos; Protótipos virtuais; Protótipos Físicos; Processos \\
CHAVES & $\begin{array}{l}\text { Paramétricos; Prototipagem Rápida; Fabricação Digital; Processo de Desenvolvimento de } \\
\text { Produto; Modelagem; Modelagem 3d; }\end{array}$
\end{tabular}

Fonte: Elaborado pelos autores

Através dessas palavras pré-estabelecidas, foi elaborada a separação de disciplinas das duas instituições considerando dois aspectos principais, sendo: a) Disciplinas que estabelecem relação direta com as tecnologias de prototipagem e fabricação digital conforme descrito nos planos de ensino e ementas; e, b) Disciplinas que estabelecem relação indireta com as tecnologias de prototipagem e fabricação digital, considerando aquelas que em seu desenvolvimento projetivo estabelecem relações inter e multidisciplinares para serem cursadas, desta forma os projetos propostos pelas mesmas, de algum modo transitarão nas tecnologias de PR e FD.

\subsection{Quadro comparativo entre os cursos de graduação em Design}

Com base nos dados obtidos apresentados, quanto às disciplinas oferecidas nas duas IES dos cursos de design, foram identificadas onze disciplinas que apresentam relação direta com as tecnologias de PR no curso da IES 1 e sete disciplinas do curso da IES 2. A tabela 2 apresenta os dados obtidos a partir da grade curricular de cada IES demonstrando o nome das disciplinas e carga horária.

Tabela 2: Comparação de disciplinas - relação direta

\begin{tabular}{|l|l|}
\hline \multicolumn{1}{|c|}{ CURSO IES 1 } & \multicolumn{1}{c|}{ CURSO IES 2 } \\
\hline Design: Materiais e Processos I-60h & Materiais e processos de produção I-60h \\
\hline Design: Materiais e Processos II - 30h & Modelos e Protótipos em Design - 90h \\
\hline Processos de Fabricação - 30h & Materiais e processos de produção II - 60h \\
\hline Ateliê de Madeira - 60h & $\begin{array}{l}\text { Técnicas de visualização e representação II - } \\
60 \mathrm{~h}\end{array}$ \\
\hline Modelagem e Prototipagem -60h & Meios e métodos de produção - 60h \\
\hline Desenho Técnico II - 90h & $\begin{array}{l}\text { Desenho paramétrico e fabricação digital - } \\
60 \mathrm{~h}\end{array}$ \\
\hline Tecnologias Assistivas - 30 & MIP: Design, materiais e produção - 90h \\
\hline Design Paramétrico e Linguagens Emergentes -60h & \\
\hline Open design e cultura maker - 60h & \\
\hline $\begin{array}{l}\text { Prototipagem e Processos de Fabricação Digital em } \\
\text { 3D -60h }\end{array}$ & \\
\hline Modelagem Avançada em 3D -60h & \\
\hline
\end{tabular}

Fonte: Elaborado pelos autores 
Como pode-se perceber, as duas instituições apresentam disciplinas variadas que se relacionam com as tecnologias de PR e FD. O curso de design da IES 1 apresenta um maior número de disciplinas que possuem relação direta com as tecnologias de prototipagem com uma carga de 600 horas ao longo dos oito semestres propostos no curso, já o curso de Design da IES 2 apresentou uma carga 480 horas ao longo de dez semestres.

Ao analisar o PPP das instituições, notou-se que algumas disciplinas, embora não apresente no plano de ensino e ementas as nomenclaturas determinadas nas palavras-chaves para a pesquisa, elas se relacionam com as tecnologias de PR e FD através da inter e multidisciplinaridade proposta por elas, ou seja, indiretamente (tabela 3 ).

Tabela 3: Comparação de disciplinas - relação indireta

\begin{tabular}{|l|l|}
\hline \multicolumn{1}{|c|}{ CURSO IES 1 } & \multicolumn{1}{c|}{ CURSO IES 2 } \\
\hline Projeto I-60h & Fundamentos do Projeto: Experimentação e Materialidade - 90h \\
\hline Projeto II - 60h & Estratégias Analógicas de Projeto - 90h \\
\hline Projeto III - 60h & Introdução ao projeto de produto - 90h \\
\hline Projeto IV - 60h & Metodologia de projeto em design - 60h \\
\hline Projeto V-90h & Projeto integrado - 180h \\
\hline Projeto VI-90h & Ergonomia I-30h \\
\hline Projeto VII - 120h & Linguagem computacional - 60h \\
\hline Projeto VIII - 150h & Ergonomia II - 30h \\
\hline Ergonomia aplicada ao Design - 60h & TCCI - 270h \\
\hline Design de Objetos - 60h & TCC II - 300 \\
\hline Design de Mobiliário - 60h & MOP: Laboratório do futuro - 90h \\
\hline Design de Ambientes - 60h & MOP: Design, espaço e ambientação - 90h \\
\hline Laboratório de Metais - 60h & \\
\hline
\end{tabular}

Fonte: Elaborado pelos autores

Conforme exposto, os números de disciplinas basicamente se equiparam, sendo treze disciplinas do curso da IES 1 e doze disciplinas do curso da IES 2, mas ao comparar a carga horária proposta pelas duas IES, é possível perceber uma grande diferença. O curso de Design da IES 1 apresenta 990 horas de disciplinas que indiretamente utilizarão no processo de desenvolvimento as tecnologias de prototipagem e fabricação digital, já o curso de Design da IES 2 apresentou um total de 1380 horas.

O figura 1, apresenta o comparativo do número de disciplinas projetivas que possuem relação direta e indiretamente com as tecnologias de PR e FD. 
Figura 1: Comparativo entre disciplinas

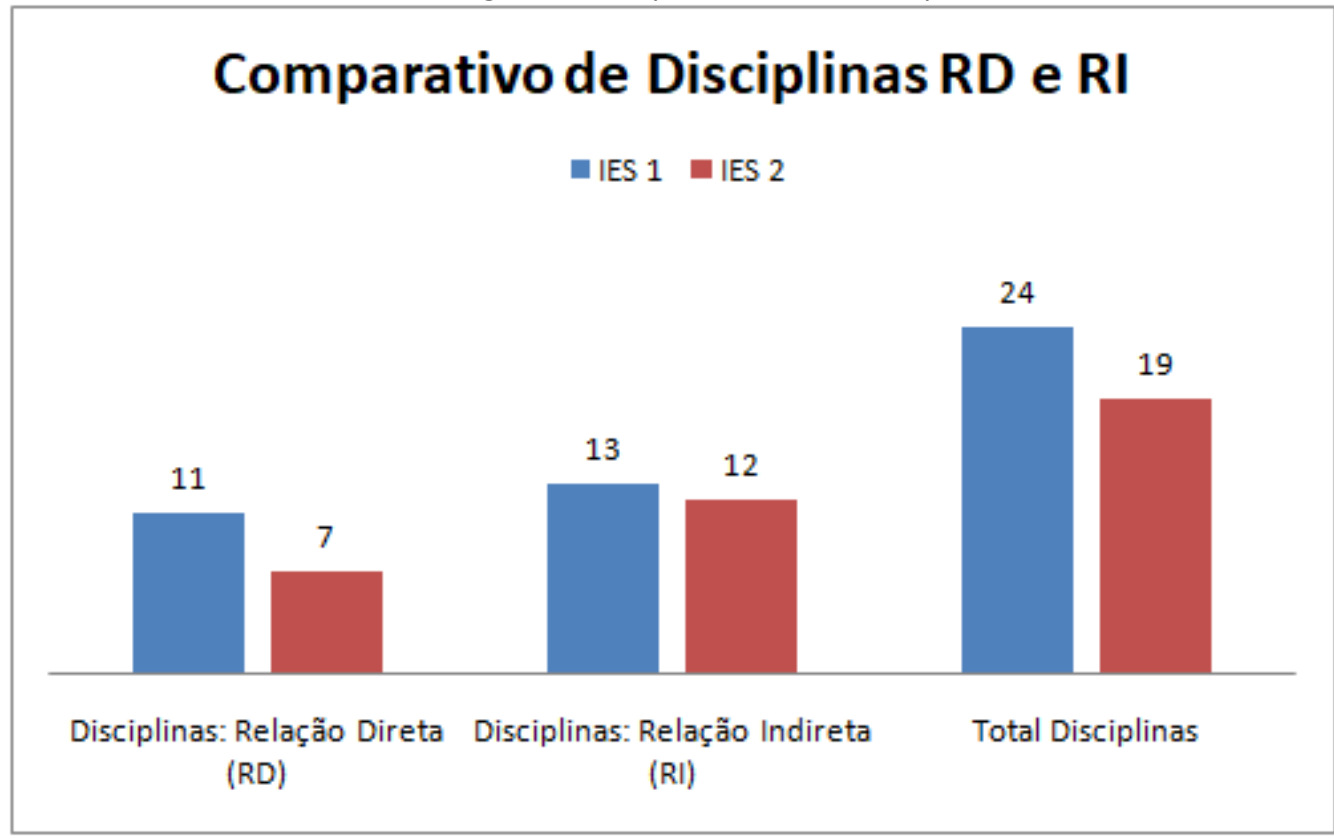

Fonte: Elaborado pelos autores

Com esses dados, é possível constatar que o curso de design da IES 1 possui um maior número de disciplinas que se relacionam com as tecnologias de prototipagem rápida e fabricação digital, já o curso da IES 2 possui uma carga horária mais expressiva (figura 2). É importante ressaltar que o curso da IES 1 possui duração de oito semestres e o da IES 2 possui dez semestres e que esta pesquisa considerou a duração total de cada curso, sendo assim, essa diferença de semestres entre as duas IES afeta diretamente a relação de disciplinas e de carga horária do curso e influenciam também na proporção comparativa de carga horária $\mathrm{x}$ relações diretas e indiretas das disciplinas.

Figura 2: Comparativo de carga horária

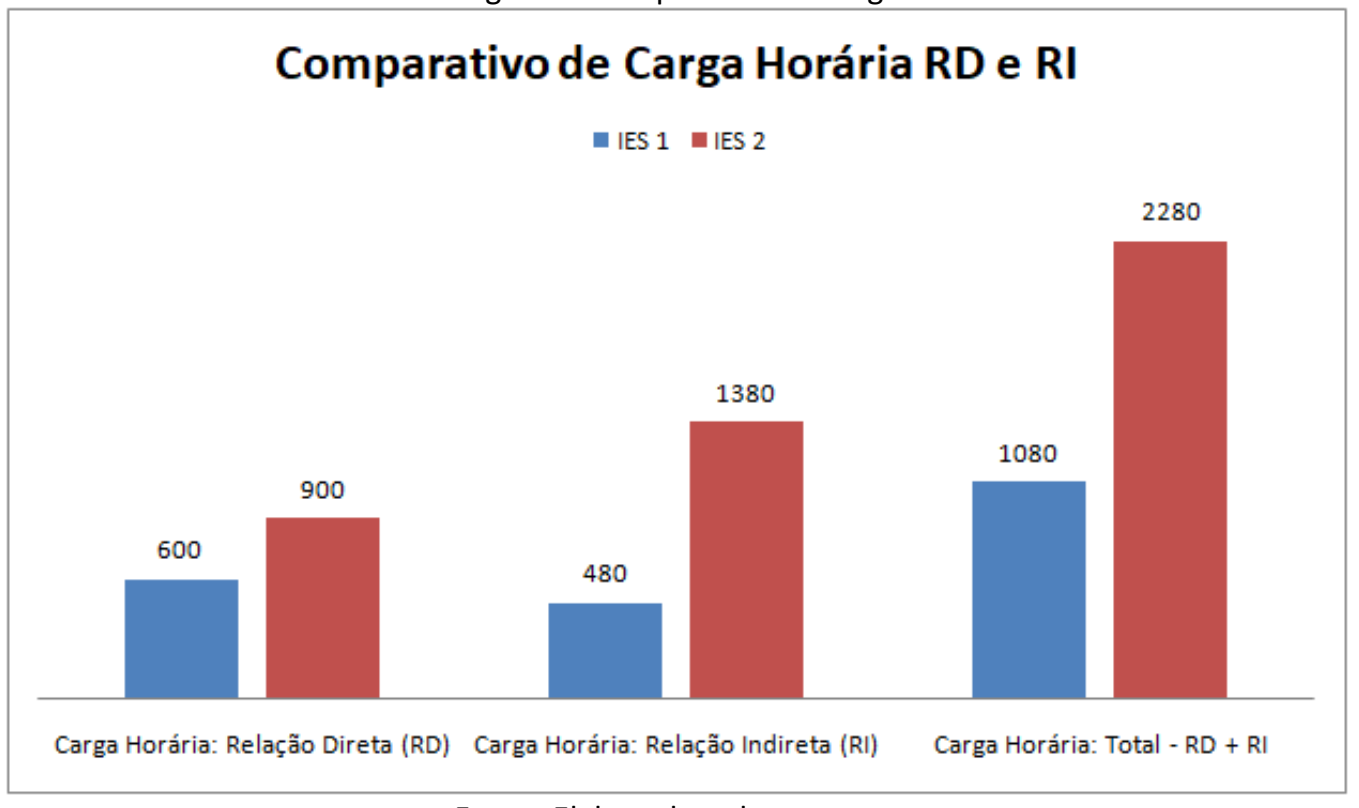

Fonte: Elaborado pelos autores 
Buscando compreender a relação da carga horária total das disciplinas que possuem Relação Direta e Relação Indireta com as tecnologias de PR, foi possível traçar um gráfico comparativo (figura 3 ) buscando entender o quanto esse valor total de RD + RI representam em porcentagem na carga horária total do curso.

Figura 3: Comparativo de carga horária projetiva e total dos cursos

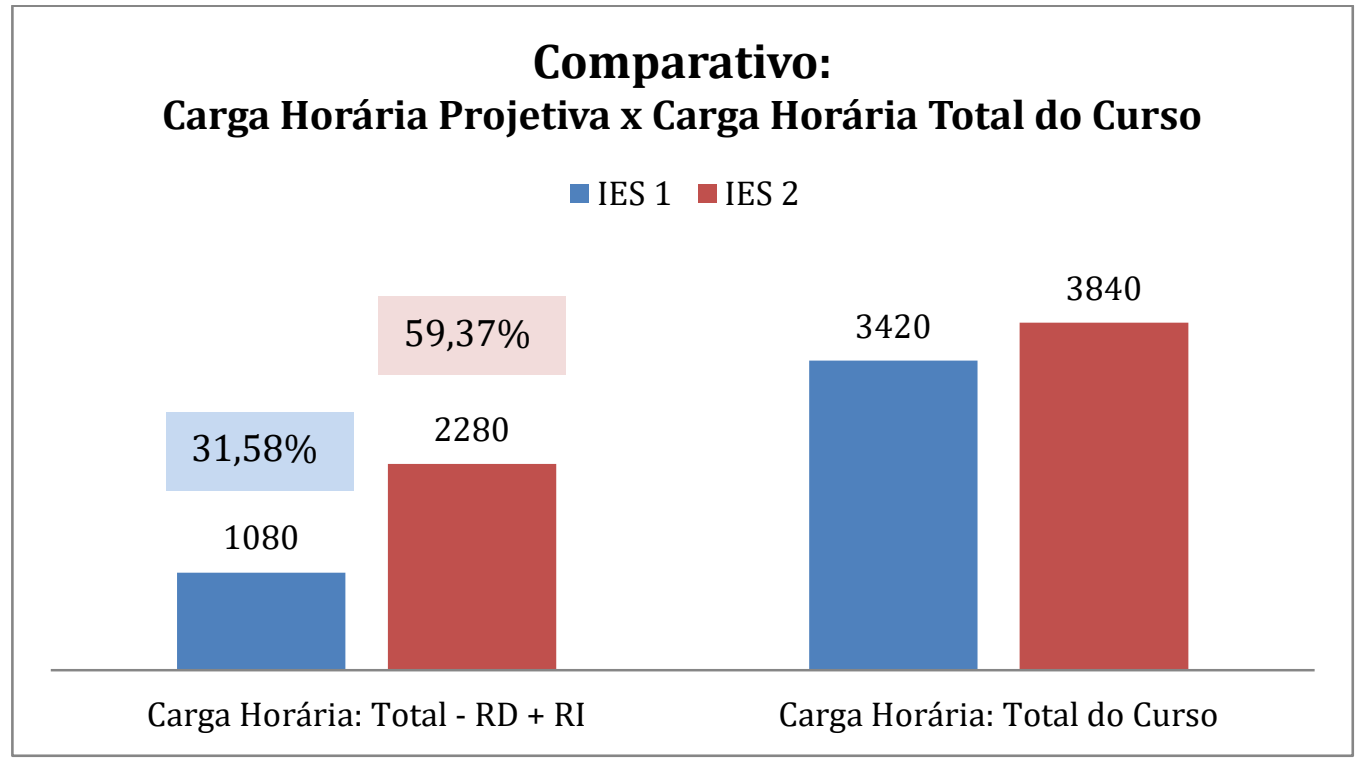

Fonte: Elaborado pelos autores

O gráfico acima demonstrou que as disciplinas projetivas que possuem relação direta e indireta com as tecnologias de prototipagem rápida e fabricação digital do curso da IES 1 representa 31,58\% da carga horária total do curso proposta ao longo de oito semestres, já o curso de design da IES 2 apresentou um total de 59,37\% ao longo de dez semestres propostos pelo curso.

\section{Conclusões}

Através das análises realizadas foi possível identificar que ambos os cursos possuem uma boa relação com as tecnologias de PR e FD permitindo ao aluno uma formação contemporânea, mais ampla e próxima da realidade do mercado de trabalho. O curso da IES 1 apresentou uma maior variedade de disciplinas que se relacionam com tais tecnologias, já o curso da IES 2 apresentou uma maior carga horária de disciplinas que se relacionam direta e indiretamente, comparando com a carga horária total do curso.

É importante enfatizar que os dados levantados neste estudo não tiveram como intuito demonstrar qual é o melhor curso, tendo em vista que para esta informação seria necessário fazer um aprofundamento maior em ambos os cursos e instituições, conhecer a fundo como é na prática a relação dessas disciplinas, prática docente, as atividades de ensino, pesquisa e extensão e do resultado os projetos dos alunos e até mesmo a contribuição deles para a sociedade.

Dado o caráter objetivo das análises realizadas e a grande quantidade de variáveis que podem influenciar diretamente no resultado do estudo, tais como: as práticas docentes, o perfil 
das ementas dos curso, a disponibilidade de tais tecnologias de PR e FD nas atividades de ensino, os materiais utilizados, a infraestrutura dos laboratórios, entre outras, por se tratar de um estudo em fase inicial, este artigo não compreende uma análise aprofundada dos cursos, para isso, está planejada realizar visitas técnicas de campo em cada IES, verificar in loco a infraestrutura dos cursos, materiais e recursos utilizados, através de questionários com docentes e discentes para entender, como o que é proposto nas ementas de disciplinas é realizado efetivamente na prática.

\section{Referências}

ALENCAR, Francisco; BÁRTOLO, Paulo Jorge. A prototipagem rápida e a digitalização ótica no design. In: Ensaios em Design: produção e diversidade. Bauru,SP: Canal 6, 2012. p. 128-150.

ASHBY, Michael; JOHNSON, Kara. Materiais e Design: arte e ciência da seleção de materiais no design de produto. 2.ed. Rio de Janeiro: Elsevier, 2011. p. 341.

BARBOSA, Ricardo Tiradentes. Design e Prototipagem: conhecimento e uso da prototipagem rápida no design brasileiro. 2009. 198 f. Dissertação (Mestrado em Design) - Universidade Estadual Paulista, Faculdade de Arquitetura, Artes e Comunicação, Bauru, SP.

BRUSCATO, Underléa Miotto; BRENDLER, Clariana Fischer; VIARO, Felipe Schneider; TEIXEIRA, Fábio Gonçalves; SILVA, Régio Pierre da; "Uso da Fabricação Digital e Prototipagem no Desenvolvimento do Projeto de Produto: Análises do Produto através de Simulações Digitais", p. 459-463 . In: Proceedings of the XVII Conference of the Iberoamerican Society of Digital Graphics: Knowledge-based Design [=Blucher Design Proceedings, v.1, n.7]. São Paulo: Blucher, 2014. ISSN 2318-6968, DOI 10.5151/despro-sigradi2013-0088

BÜRDEK, Bernhard E. Diseño: Historia, teoria y práctica del diseño industrial. Barcelona: Gustavo Gili, 1994

GROOVER, Mikel P. Introdução aos processos de fabricação. Tradução Anna Clara Araujo; tradução e revisão técnica André Ribeiro de Oliveira ...[et al.] - 1. ed. Rio de Janeiro: LTC, 2014.

LAKATOS, Eva Maria; MARCONI, Marina de Andrade. Fundamentos da metodologia científica. 4. ed. São Paulo: Atlas, 2001.

LESKO, Jim. Design Industrial: Guia de Materiais e Fabricação. 2. ed. São Paulo: Blucher, 2012. P. 338-340.

LÖBACH, Bernd. Design Industrial: Bases para configuração dos produtos industriais. São Paulo: Blucher, 2001, pp. 141.

MOURA, Mônica. Design, Arte e Tecnologia. In: Design, Arte e Tecnologia. Coletânea de artigos e projetos experimentais. São Paulo: Editora Rosari, 2005. CD-ROM.

PORTUGAL, Cristina. Design, Educação e Tecnologia. 1. ed. Rio de Janeiro: Rio Books, 2013. 
VOLPATO, Néri. Prototipagem Rápida: Tecnologias e Aplicações. São Paulo: Edgard Blucher,2007.

SALVAN PAGNAN, Caroline; MOTTIN, Artur Caron. Cuad. Cent. Estud. Diseñ. Comun., Ensayos, Ciudad Autónoma de Buenos Aires, n. 69, p. 1-6, sept. 2018 .Disponivel em <http://www.scielo.org.ar/scielo.php?script=sci_arttext\&pid=S1853-

35232018000400009\&lng=es\&nrm=iso>. Acesso em: 24 mar 2018. 